\title{
Extended quasilocal Thermodynamics of Schwarzchild-anti de Sitter black holes
}

\author{
W. B. Fontana ${ }^{1, *}$, M. C. Baldiotti ${ }^{1, \dagger}$, R. Fresneda ${ }^{2}$, and C. Molina ${ }^{3}$ \\ ${ }^{1}$ Departamento de Física, Universidade Estadual de Londrina, \\ 86051-990, Londrina-PR, Brazil. \\ ${ }^{2}$ Centro de Matemática, Computação e Cognição, Universidade \\ Federal do ABC, Av. dos Estados 5001, 09210-580, Santo Andr-SP, \\ Brazil. \\ ${ }^{3}$ Escola de Artes, Ciências e Humanidades, Universidade de São \\ Paulo, Av. Arlindo Bettio 1000, CEP 03828-000, São Paulo-SP, \\ Brazil. \\ Electronic Adress: *weslei@uel.br; †baldiotti@uel.br; \\ ${ }^{2}$ rodrigo.fresneda@ufabc.edu.br; ${ }^{3}$ cmolina@usp.br
}

\begin{abstract}
In this work we study a homogeneous and quasilocal Thermodynamics associated to the Schwarzschild-anti de Sitter black hole. The usual thermodynamic description is extended within a Hamiltonian approach with the introduction of the cosmological constant in the thermodynamic phase space. The macroscopic treatment presented is consistent in as much as it respects the laws of black hole Thermodynamics and accepts the introduction of any thermodynamic potential. We are able to construct new equations of state that characterize the Thermodynamics. Novel phenomena can be expected from the proposed setup.
\end{abstract}

\section{Introduction}

Over the last few decades, research on black hole Physics has suggested fundamental connections between Gravity, Thermodynamics and Quantum Field Theory [1]. Theoretical evidences indicate that black hole Thermodynamics is a key to this relationship, dictating the laws which black holes obey and, at the same time, showing that those laws can be seen as a thermodynamic description of gravitational systems [1, 2, 3.

Since the pioneering work of Bekenstein [4, it is well-known that black holes possess an entropy proportional to their horizon area. Afterwards Hawking, Carter and Bardeen provided a general proof of the laws of black hole Mechanics 
[5]. A genuine black hole Thermodynamics was derived from Hawking's result of black hole evaporation [6], which showed that black holes emit radiation due to semiclassical effects, and thus have an associated physical temperature. Since then, black hole Thermodynamics has become a major topic in Physics, with special attention to the thermodynamic properties of asymptotically anti de Sitter (AdS) geometries (see for example [7, 8]) due to the developments in anti de Sitter/conformal field theories (AdS/CFT) correspondences [9, 10, 11].

In the present work we focus on the thermodynamic behavior of $D$-dimensional Schwarzchild-anti de Sitter black holes (SAdS). We consider a macroscopic setup which arises from a description where the SAdS black hole is in equilibrium with a thermal atmosphere that is generated by Hawking emission 77. We will refer to minimal SAdS Thermodynamics as the description where there is only one thermodynamic variable, the entropy (or the horizon radius). In such a scenario, there is no distinction between isothermic and isentropic processes, and the notion of a thermodynamic temperature is not well-defined 12. Besides, in this minimal setup, the first law of Thermodynamics is not consistent with the Smarr formula [13. Therefore, the minimal description is not a thermodynamic theory in the standard sense. These issues can be fixed once we extend the thermodynamic theory in order to have additional degrees of freedom. So, in the case of a SAdS black hole, this amounts to a Thermodynamics which is closer to that of usual matter systems [14.

The extension of the SAdS black hole Thermodynamics, obtained by introducing the cosmological constant as a dynamical variable, furnishes a good path towards a consistent theory. In this formalism $\Lambda$ is interpreted as a pressure and the black hole mass as the enthalpy, instead of the internal energy [15]. The cosmological constant is needed as a thermodynamic parameter for the theory to have homogeneous equations of state. Homogeneity is required for extensivity [13] and for the existence of an integrating factor for the reversible heat exchange [16]. However, homogeneity requires that $\Lambda$ must be introduced in the theory in a very specific manner, otherwise inconsistencies appear (for example, in the construction of the thermodynamic potentials, as pointed out in [17]). In particular, if one tries to use the cosmological constant as a thermodynamic variable in this extension, the obtained theory will present singularities in the Legendre transformation between $\Lambda$ and its conjugate variable [18].

Even after the construction of a consistent theory, there is a problem related to the physical interpretation of the thermodynamic temperature. The usual definition of black hole temperature is based on the horizon surface gravity. But this quantity is not uniquely defined in stationary asymptotically anti de Sitter geometries, since there is no preferred normalization for the associated time-like Killing vector field.

An attempt to solve this ambiguity problem was proposed in [19, 20. The authors assumed a quasilocal approach, where physical quantities are defined on a bounded spacetime region $R$. In this way the time-like Killing vector field has no divergences and it is well-defined. This procedure defines new quantities on the boundary, such as an energy function $E$ that plays the role of the internal energy in the thermodynamic description. As one is dealing with 
a bounded region, the quasilocal approach introduces a new thermodynamic variable connected with the position $R$ of the observer in spacetime. As a result, the temperature function depends on this new variable. In this formulation, the temperature is redshifted to zero as $R \rightarrow \infty$ due to the associated Tolman factor [21, which can be interpreted as a manifestation of the confining character of the AdS asymptotics. Although the quasilocal Thermodynamics gives a better definition of the temperature in a non-asymptotic flat spacetime, it still lacks homogeneity, and all related problems remain.

In a previous work [17], we extend the minimal setup using a Hamiltonian approach to Thermodynamics [22, where the equations of state are realized as constraints on a symplectic phase space (see also [23] for an approach based on the Hamilton-Jacobi formalism). We are able to incorporate $\Lambda$ in a consistent manner in [17, solving the homogeneity issue and obtaining a sensible Smarr formula for the SAdS Thermodynamics. However, we are not able to fix the functional form of $\Lambda$ in [17, as it remains dependent on free parameters of the model. In this work we construct an extended version for the quasilocal thermodynamic description using the aforementioned Hamiltonian method. The approach is to construct a macroscopic effective description where semiclassical effects are implicitly assumed, although no microscopic formalism (e.g. quantum field theories) is used. The resulting theory has none of the issues described above and gives a well-defined interpretation for the thermodynamic temperature. We show that in this formalism it is possible to promote $\Lambda$ to a thermodynamic variable. Besides, using geometric and thermodynamic arguments, we propose some functional forms for the cosmological constant.

The structure of this work is as follows. In section 2 we review the minimal description of the $D$-dimensional SAdS black hole. In section 3 we give a brief account of the extensions which use the cosmological constant as a thermodynamic variable. In section 4 we discuss the quasilocal approach to black hole Thermodynamics. In section 5 the quasilocal thermodynamic description is extended by means of the Hamiltonian approach. In the process, a new equation of state for the black hole is constructed. Stability issues are studied in section 6. In section 7, we discuss the final considerations for this work and some paths that can be followed in the future. Throughout this paper, we use the metric signature $(-++\cdots+)$ and natural units with $G=\hbar=c=k_{B}=1$.

\section{Minimal Schwarzchild-AdS Thermodynamics}

The Schwarzchild-anti de Sitter spacetime is the spherically symmetric vacuum solution of the Einstein's equations with a negative cosmological constant $\Lambda$. Its metric can be written as

$$
d s^{2}=-N^{2}(r) d t^{2}+\frac{1}{N^{2}(r)} d r^{2}+r^{2} d \Omega_{D-2}^{2},
$$

where $d \Omega_{D-2}^{2}$ is the squared line element of the $(D-2)$-dimensional sphere $S^{D-2}$. The lapse function $N(r)$ in (1) is expressed in terms of the (negative) 
cosmological constant $\Lambda$ and a (positive) parameter $M$ as

$$
N^{2}(r)=1-\frac{16 \pi M}{(D-2) B_{D}} \frac{1}{r^{D-3}}-\frac{2 \Lambda r^{2}}{(D-1)(D-2)},
$$

where $B_{D}$ denotes the canonical volume of $S^{D-2}$, namely,

$$
B_{D}=\frac{2 \pi^{\frac{D-1}{2}}}{\Gamma\left(\frac{D-1}{2}\right)} .
$$

It is convenient to define an effective cosmological constant $\tilde{\Lambda}$ that is related to $\Lambda$ as

$$
\tilde{\Lambda} \equiv \frac{2 \Lambda}{(D-1)(D-2)} .
$$

Staticity of SAdS spacetime is characterized by the existence of a global Killing vector field $\partial / \partial t$, which is time-like outside the black hole. However, since the geometry is not asymptotically flat, there is no preferred normalization for the Killing field. In fact, its norm

$$
\left|g\left(\frac{\partial}{\partial t}, \frac{\partial}{\partial t}\right)\right|=N^{2}(r)
$$

diverges as $r \rightarrow \infty$.

The Schwarzchild-anti de Sitter spacetime has a Killing horizon at $r=r_{+}$ associated to $\partial / \partial t$. The horizon radius $r_{+}$is the unique positive root of $N(r)$. A surface gravity $\kappa$ and a surface area $A$, or more precisely a $(D-2)$-volume, can be associated to the horizon, whose expressions in terms of $r_{+}$are

$$
\kappa=\frac{1}{2}\left[\frac{D-3}{r_{+}}-(D-1) \tilde{\Lambda} r_{+}\right], A=B_{D} r_{+}^{D-2} .
$$

Using the expressions (6), one obtains the following relation among the parameters $M, A$ and $\kappa$,

$$
8 \pi \frac{D-1}{D-2} M=A\left(\frac{A}{B_{D}}\right)^{\frac{1}{D-2}}+\kappa A .
$$

The existence of a Killing horizon implies a thermodynamic description for the Schwarzchild-anti de Sitter spacetime [24. We will apply methods of classical mechanics and thermodynamics on an effective theory, where it is implicitly assumed that semiclassical effects generate a thermal Hawking atmosphere [7, 17, 25] around the black hole, and that it is in thermal equilibrium with its atmosphere. In the minimal description, there is only one thermodynamic variable: the entropy $S$. Hence, the fundamental equation has the form $U=U(S)$, where $U$ denotes the system's internal energy. Concretely, the minimal Schwarzchild-anti de Sitter Thermodynamics is defined characterizing the internal energy $U$, the entropy $S$ and the temperature $T$ as

$$
U \equiv M, \quad S \equiv \frac{A}{4}, \quad T=\frac{\kappa}{2 \pi} .
$$


In fact, using the construction (8), one verifies that

$$
\frac{\partial U}{\partial S}=T \Rightarrow d U=T d S,
$$

which is the first law of Thermodynamics in the minimal scenario.

Relation (7) can be rewritten in terms of the thermodynamic variables $U, S$ and $T$ as

$$
\frac{D-1}{D-2} U-T S=\frac{1}{2 \pi}\left(\frac{B_{D}}{4} S^{D-3}\right)^{\frac{1}{D-2}} .
$$

The above expression is the only equation of state in the minimal thermodynamic description of SAdS black holes.

Despite its simplicity, minimal SAdS Thermodynamics has significant drawbacks. For $\Lambda=0$ one can fix the normalization (5) such that $g(\partial / \partial t, \partial / \partial t)=-1$ at the spatial infinity and the obtained theory is homogeneous. While, for the SAdS spacetime, there is no preferred normalization for the time-like Killing field. Thus, there is no unique definition of temperature, as a Killing field proportional to $\partial / \partial t$, will have a surface gravity different from $\kappa$ in (6). Also, for $\Lambda \neq 0$, the internal energy is no longer homogeneous and the minimal Thermodynamics is not standard [16, 17].

\section{The cosmological constant as a thermodynamic variable}

In this subsection we review the first attempts at generalizing the minimal AdS Thermodynamics. The possibility of treating the cosmological constant as a dynamical variable was proposed in [26, 27, by coupling the gravitational field to tensor fields. A quasilocal treatment was developed in [28], by considering an Einstein-Hilbert-Dilaton action coupled to various types of Abelian and non-Abelian gauge fields. These works do not address the problem of the conjugate variable to $\Lambda$ in a thermodynamic interpretation, neither do they consider different thermodynamic descriptions, which can be obtained by Legendre transformations of $\Lambda$ and its conjugated pair.

The internal energy $M$ in the minimal AdS Thermodynamics (as defined in Section 2) is not a first order homogeneous function. In the asymptotically flat case, Euler's theorem for homogeneous functions provides a route between the first law of black hole mechanics and the Smarr formula [29] for stationary black holes [30, 31]. However, when $\Lambda \neq 0$, in order to apply this procedure, one must take into account the scaling properties of the cosmological constant [32]. By direct calculation for specific (A)dS black hole solutions, expressions for the Smarr formula and the first law with a variable $\Lambda$ have been obtained in [32, 33, 34, 35, 36]. The first law can also be obtained using Hamiltonian perturbation theory techniques [37, 38, 39]. Besides the scaling argument [13], homogeneity of the equations of state is required for a consistent black hole Thermodynamics [16, 40]. 
Using the Killing potential introduced in [41, 42], and the techniques introduced in [33], it is possible to extend the Smarr formula to the $D$-dimensional SAdS scenario. It follows that, for the case of the AdS black hole with no charge nor angular momentum, one has the $D$-dimensional Smarr formula [43,

$$
(D-3) M=(D-2) \frac{\kappa A}{8 \pi}+2 \frac{\theta \Lambda}{8 \pi} .
$$

In expression (11), $\theta$ represents a new thermodynamic variable, conjugated to $\Lambda$. Using (6), (7) and the Smarr formula (11) for $D$ dimensions, one obtains

$$
\theta=\frac{B_{D}}{D-1}\left(\frac{4 S}{B_{D}}\right)^{\frac{D-1}{D-2}}=\frac{B_{D}}{D-1} r_{+}^{D-1} .
$$

Result (12) identifies $\theta$ with the "volume extracted from spacetime" by the black hole [13]. This identification suggests that the cosmological constant $\Lambda$ should be interpreted as a pressure and the black hole mass $M$ should be identified with its enthalpy $H$ [13]. In this context, there are two independent thermodynamic variables, such that

$$
H \equiv M, S \equiv \frac{A}{4}, T \equiv \frac{\kappa}{2 \pi}, \Pi \equiv-\frac{\Lambda}{8 \pi},
$$

where $\Pi$ is the thermodynamic pressure associated to $\theta$. A different approach was proposed in [4], where conserved charges in AdS were analyzed. When the cosmological constant is considered as a thermodynamic variable, new phenomena appear in the description of black holes. For example, these objects behave like a Van der Waals fluid, with properties sometimes encountered in more commonplace scenarios [45]. One often calls black hole chemistry the setup where $\Lambda$ is a thermodynamic variable [46, 47] (see [8] for a review).

Despite the intriguing arguments defining $M$ as the enthalpy, there is an inconsistency in the procedure. The problem is due to the fact that $\theta$ does not depend on $\Pi$, which leads to a singularity in the Legendre transformation of the pair $(\Pi, \theta)$. As a matter of fact, from (13) one sees that

$$
\left.\frac{\partial H}{\partial \Pi}\right|_{S}=\theta .
$$

Considering now (11) and (7), one determines the internal energy $U$,

$$
U=H-\Pi \theta=(D-2) \frac{B_{D-2}}{16 \pi}\left(\frac{4 S}{B_{D-2}}\right)^{\frac{D-3}{D-2}},
$$

and it follows that (15) does not give the first law, that is,

$$
\frac{\kappa}{2 \pi}=T \neq \frac{\partial U}{\partial S} .
$$

We stress that this problem exists only when the black hole possesses no other characteristic besides its mass. When charge or angular momentum are present, 
the thermodynamic potential can be constructed by thermodynamic [18] or statistical mechanics arguments 32, and no singularity is present. In the present work we will consider spherically symmetric and electrically neutral spacetimes. For this class of black holes, we will construct a singularity-free thermodynamic description.

\section{Quasilocal Schwarzchild-AdS Thermodynamics}

To solve some of the problems of the minimal Thermodynamics, one possible approach is a quasilocal treatment, where the quantities of interest are defined in a bounded region. More specifically, we will use the general formalism presented by Brown and York 20, which was later applied to asymptotic anti de Sitter geometries by Brown, Creighton and Mann [19. It is worth mentioning other approaches based on extensions of the Noether theorem, presented for instance in 48] and references therein.

In the Brown-York approach, one considers a bounded region $\mathcal{M} \subset \mathcal{S}$ of a spacetime $\mathcal{S}$ with (pseudo-Riemannian) metric $g_{\mu \nu}$ in $D$ dimensions. It is assumed that $\mathcal{M}$ has the topology $\Sigma \times I$, where $\Sigma$ is a space-like $(D-1)$ dimensional hypersurface and $I$ is an interval of the real line. The region $\mathcal{M}$ is composed by three pieces: "initial" and "final" space-like hypersurfaces $\mathcal{T}_{I}$ and $\mathcal{T}_{F}$; and a hypersurface $\mathcal{B}$ which connects $\mathcal{T}_{I}$ and $\mathcal{T}_{F}$. It follows that $\mathcal{B}=B \times I$, where $B$ is a compact $(D-2)$-dimensional space-like surface. That is, $\mathcal{B}$ is foliated into $(D-2)$-surfaces. The induced metric on $B$ is denoted by $\sigma_{a b}$. Neither global hyperbolicity nor specific asymptotic structures are assumed for $\mathcal{S}$.

Brown and York employed a Hamilton-Jacobi analysis of the action functional describing Einstein's gravity in spacetimes with finite boundaries. The appropriate gravitational action for this description is 49.

$$
S_{g}=S_{0}+\frac{1}{\alpha} S_{L}
$$

with $\alpha$ being a coupling constant and the action $S_{L}$ being given by

$$
\begin{array}{rl}
S_{L}=\int_{\mathcal{M}} d^{D} & x \sqrt{-g}\left(\frac{\mathcal{R}}{2}-\Lambda\right)+\int_{\mathcal{T}_{F}} d^{D-1} x \sqrt{h^{F}} K^{F} \\
& -\int_{\mathcal{T}_{I}} d^{D-1} x \sqrt{h^{I}} K^{I}-\int_{\mathcal{B}} d^{D-1} \sqrt{-\gamma} \Theta .
\end{array}
$$

In (17), $S_{0}$ is a functional of the metric on $\partial \mathcal{M}$ such that $\delta S_{0}$ vanishes when the metric is fixed on the boundary $\partial \mathcal{M}$. The induced metrics on $\mathcal{T}_{I}, \mathcal{T}_{F}$ and $\mathcal{B}$ are denoted by $h_{i j}^{I}, h_{i j}^{F}$ and $\gamma_{i j}$ respectively. The curvature scalar on $\mathcal{M}$ is denoted by $\mathcal{R}$. The quantities $K^{I}, K^{F}$ and $\Theta$ are the traces of the extrinsic curvatures in the hypersurfaces $\mathcal{T}_{I}, \mathcal{T}_{F}$ and $\mathcal{B}$ respectively.

Decomposing the metric $\gamma_{i j}$ in the ADM form [50], one obtains that

$$
\gamma_{i j} d x^{i} d x^{j}=-N^{2} d t^{2}+\sigma_{a b}\left(d x^{a}+V^{a} d t\right)\left(d x^{b}+V^{b} d t\right)
$$


where $N$ is the lapse function and $V^{a}$ is the shift vector. In the Brown-York approach, the main contribution from the variation of $S_{g}$ comes from the boundary $\mathcal{B}$. Denoting $u_{i}$ as the unit normal vector to $B$ and $\sigma_{i}^{a}=\delta_{i}^{a}$ the tensor that projects covariant tensors from $\mathcal{B}$ to $B$, it follows that

$$
\left.\delta S_{g}\right|_{\mathcal{B}}=\int_{\mathcal{B}} d^{D-1} x \sqrt{\sigma}\left[j_{a} \delta V^{a}-\varepsilon \delta N+\left(\frac{N}{2}\right) s^{a b} \delta \sigma_{a b}\right],
$$

where

$$
\begin{aligned}
\varepsilon & =\frac{1}{\alpha} k-\varepsilon_{0}, j_{i}=-\frac{2}{\sqrt{h}} \sigma_{i j} P^{j k} n_{k}-\left(j_{0}\right)_{i}, \\
s^{a b} & =\frac{1}{\alpha}\left(k^{a b}+\left(n_{\mu} a^{\mu}-k\right) \sigma^{a b}\right)-\left(s_{0}\right)^{a b} .
\end{aligned}
$$

In expressions (20)-(22), $n_{\mu}$ represents the components of the unit normal to $\mathcal{B}$, $k^{a b}$ is the extrinsic curvature of the boundary $B$ and $k$ its trace, $P^{i j}$ denotes the gravitational momentum for the hypersurface $\Sigma$ and $a^{\mu}=u^{\nu} \nabla_{\nu} u^{\mu}$ indicating the acceleration of $u^{\mu}$. The terms with index zero in (21) and (22) are proportional to the functional derivatives of $S_{0}$.

From (20), it follows that the term $-\sqrt{\sigma} \varepsilon$ is equal to the time rate of change of the action, wherein the lapse function controls the changes of $\mathcal{B}$ with respect to time. Thus, $\varepsilon$ is identified as an energy surface density and the total quasilocal energy is defined by integration over the $(D-2)$-dimensional surface $B$ [20],

$$
E=\int_{B} d^{D-2} x \sqrt{\sigma} \varepsilon
$$

Let us analyze the $D$-dimensional Schwarzchild-anti de Sitter black hole. In $D$ dimensions, we set the coupling constant to $\alpha=8 \pi$. Since the geometry is spherically symmetric, it is natural to consider the surfaces $B$ as $(D-2)$-spheres, specified by $r=R$, with constant $R$. Staticity of the SAdS geometry allows the identification of $\mathcal{T}_{I}$ and $\mathcal{T}_{F}$ with the hypersurfaces $t=t_{I}$ and $t=t_{F}$. Given the choices made, the energy density $\varepsilon$ associated to the surface $r=R$ is

$$
\varepsilon=-\frac{D-2}{8 \pi R} \sqrt{1-\frac{16 \pi M}{(D-2) B_{D} R^{D-3}}-\tilde{\Lambda} R^{2}}-\varepsilon_{0}(R) .
$$

Integrating over $B$, one obtains the total energy:

$$
E=-R^{D-2} B_{D}\left[\frac{D-2}{8 \pi R} \sqrt{1-\frac{16 \pi M}{(D-2) B_{D} R^{D-3}}-\tilde{\Lambda} R^{2}}+\varepsilon_{0}(R)\right] .
$$

We note that some quantities, such as $E$ in (25), depend on the arbitrary function $\varepsilon_{0}$.

Brown, Creighton and Mann [19] assumed that the quantity $E$ in (25) represents the internal energy of the thermodynamic system. Following [19], the 
surface pressure $P$ at the boundary $r=R$ can be obtained for the $D$-dimensional Schwarzschild-anti de Sitter spacetime:

$$
\begin{aligned}
P & \equiv-\frac{\partial E}{\partial\left(B_{D} R^{D-2}\right)} \\
& =\frac{R(D-3)}{8 \pi N(R)}\left[R^{-2}-\frac{8 \pi M R^{1-D}}{(D-2) B_{D}}-\frac{D-2}{D-3} \tilde{\Lambda}\right]+\frac{\partial\left(R^{D-2} \varepsilon_{0}\right)}{\partial\left(R^{D-2}\right)} .
\end{aligned}
$$

In the same fashion, a local temperature $T_{L}$ can be found as

$$
T_{L} \equiv \frac{\partial E}{\partial S}=\frac{D-3}{4 \pi N(R)}\left[\left(\frac{4 S}{B_{D}}\right)^{-\frac{1}{D-2}}-\frac{D-1}{D-3} \tilde{\Lambda}\left(\frac{4 S}{B_{D}}\right)^{\frac{1}{D-2}}\right]
$$

The local temperature $T_{L}$ in (27) is damped by a term proportional to the inverse of the lapse function

$$
T_{L}=\frac{1}{N(R)} \frac{\kappa}{2 \pi}=\frac{T}{N(R)} .
$$

The damping of a local temperature by the Tolman factor, as observed by Brown, Creighton and Mann in their approach, is a generic characteristic of any reasonable Thermodynamics in a static spacetime [21].

Thus, in the quasilocal description of Schwarzschild-anti de Sitter spacetime, the temperature measured by an observer at $r=R$ is a well-defined quantity. Also, the Thermodynamics is constructed with two degrees of freedom, since the position $R$ is introduced as a new thermodynamic variable. Two of the problematic issues in the minimal SAdS thermodynamic description are solved.

Still, the problem associated to homogeneity remains in the SAdS Thermodynamics proposed by Brown, Creighton and Mann. Indeed, the lapse function $N(r)$ must be a homogeneous function of order zero. This will be true only if $M / R^{D-3}$ and $\Lambda R^{2}$ also are homogeneous functions of order zero, as it is apparent from (2). In order to recover homogeneity, $\Lambda$ will be considered a thermodynamic parameter with the help of the formalism developed in [17, 22].

\section{Extended quasilocal SAdS Thermodynamics}

\subsection{Extended phase space}

The Hamiltonian approach to Thermodynamics consists of the identification of thermodynamic variables with local coordinates $(q, p)$ of a phase space. In this manifold, a Hamilton function $h(q, p, t)$ is introduced, and the symplectic structure is given by $\omega=d \theta$, where $\theta=p d q$ is the tautological one-form. Equations of state are realized as constraints and the one-form $\theta$ on the surface of constraints becomes the differential of a thermodynamic potential. In this way, one shows that Legendre transformations of thermodynamic potentials amount to canonical transformations in phase space. Furthermore, one can extend this 
description by the addition of new coordinates and momenta $(\tau, \xi)$. This is done without increasing the number of physical degrees of freedom, by supplying a Hamiltonian constraint $H=\xi+h(q, p, \tau)$. As a result, the tautological oneform $\theta$ becomes the Poincaré-Cartan form $p d q-h d t$ on the constraint surface. For further details, see [22].

Let us first rename the thermodynamic variables as

$$
q=\frac{4 S}{B_{D}}, \quad p=\pi T_{L}=\frac{\kappa}{2 N}, \quad x=R^{D-2}, \quad \varpi=-4 \pi P .
$$

They label local coordinates on an open region of $\mathbb{R}^{4}$ with symplectic structure given by the tautological one-form $\theta=p d q+\varpi d x$. The equations of state (26) and (27) become constraint equations $\phi_{1}=0$ and $\phi_{2}=0$, which are written in terms of the mechanical variables (29) as

$$
\begin{aligned}
\phi_{1} & =p-\frac{D-3}{4 N}\left(q^{-\frac{1}{D-2}}-\frac{D-1}{D-3} q^{\frac{1}{D-2}} \tilde{\Lambda}\right) \\
\phi_{2} & =\frac{D-3}{4 N}\left\{2 x^{-\frac{1}{D-2}}+x^{-1}\left[\left(q^{\frac{D-1}{D-2}}-2 \frac{D-2}{D-3} x^{\frac{D-1}{D-2}}\right) \tilde{\Lambda}-q^{\frac{D-3}{D-2}}\right]\right\} \\
& +\varpi+4 \pi \frac{\partial\left(x \varepsilon_{0}\right)}{\partial x} .
\end{aligned}
$$

We will consider the energy (25) a thermodynamic potential, so one has a fundamental equation. Then, the form $\theta$ is the differential $d E$ on the constraint surface $\phi_{1}=\phi_{2}=0,\left.\theta\right|_{\phi_{1}=\phi_{2}=0}=d E$. The symplectic structure in the phase space $(q, p ; x, \varpi)$ is defined by the two-form $\omega=d \theta$. According to the canonical Poisson structure given by $\omega$, the Poisson brackets between the constraints vanish identically, so the set of constraints is first-class. Therefore, there are no physical degrees of freedom, as expected from the general theory [22]. We shall extend the description given in section 4 by introducing a new canonical pair $(\xi, \tau)$ of mechanical variables, such that the tautological one-form $\theta$ becomes

$$
\theta \mapsto \tilde{\theta}=\frac{B_{D}}{4 \pi}(p d q+\varpi d x)+\xi d \tau
$$

with $\xi=\partial E / \partial \tau$ on the constraint surface. We follow [17] in that $\Lambda$ is considered a function on phase space, depending on the coordinates $q, x$ and $\tau$. In particular, $\varepsilon_{0}$ and $N$ depend on these coordinates through $\Lambda$. Since the tautological one-form $\tilde{\theta}$ must reproduce the differential $d E$ on the constraint surface, one is led to a new constraint,

$$
\begin{aligned}
\phi_{3} & =\xi-\frac{\partial E}{\partial \tau} \\
& =\xi+\frac{B_{D}(D-2)}{16 N \pi}\left(q^{\frac{D-1}{D-2}}-x^{\frac{D-1}{D-2}}\right) \frac{\partial \tilde{\Lambda}}{\partial \tau}+x B_{D} \frac{\partial \varepsilon_{0}}{\partial \tau} .
\end{aligned}
$$

With this, the notion of thermodynamic energy is not the same as the one in the original theory. In addition, the constraints (30) receive contributions coming 
from derivatives of $\Lambda$ and $\varepsilon_{0}$ with respect to the coordinates, which in terms of thermodynamic quantities are described by

$$
\begin{aligned}
& P \mapsto P+\frac{D-2}{16 \pi N}\left[\left(\frac{4 S}{B_{D}}\right)^{\frac{D-1}{D-2}}-R^{D-1}\right] \frac{\partial \tilde{\Lambda}}{\partial\left(R^{D-2}\right)}, \\
& T_{L} \mapsto T_{L}-B_{D} \frac{D-2}{16 \pi N}\left[\left(\frac{4 S}{B_{D}}\right)^{\frac{D-1}{D-2}}-R^{D-1}\right] \frac{\partial \tilde{\Lambda}}{\partial S}-B_{D} R^{D-2} \frac{\partial \varepsilon_{0}}{\partial \tilde{\Lambda}} \frac{\partial \tilde{\Lambda}}{\partial S} .
\end{aligned}
$$

The total set of constraints is again first-class, resulting in a theory with no physical degrees of freedom. We note that if the cosmological constant depends on the entropy, the temperature $T_{L}$ no longer has the usual geometric interpretation as surface gravity.

Now we impose homogeneity to the equations of state, which is a necessary condition in order to have a consistent black hole Thermodynamics [16, 40]. Although homogeneity does not completely fix the functional form of $\Lambda$, it does impose strong conditions on it. Considering the extensivity of the entropy $S \rightarrow \lambda S$ in (27), one has

$$
T_{L} \rightarrow \lambda^{-\frac{1}{D-2}} T_{L}
$$

and, since the lapse function (22) is homogeneous of order zero, we obtain

$$
\begin{aligned}
R & \rightarrow \lambda^{\frac{1}{D-2}} R, \quad E \rightarrow \lambda^{\frac{D-3}{D-2}} E, \quad P \rightarrow \lambda^{-\frac{1}{D-2}} P, \\
\tilde{\Lambda} & \rightarrow \lambda^{-\frac{2}{D-2}} \tilde{\Lambda}, \quad \xi \rightarrow \lambda^{\frac{D-3}{D-2}-c} \xi, \quad \tau \rightarrow \lambda^{c} \tau .
\end{aligned}
$$

Once the lapse function $N$ does not depends explicitly on $\tau$, but only through $\Lambda(S, R, \tau)$, it is not possible to fix the homogeneity order of $\tau$ by demanding that $N$ be homogeneous of order zero. So, $c$ remains an arbitrary real constant. This constant connects all the thermodynamic descriptions that respect the homogeneity condition.

Consider the Euler theorem for homogeneous functions [13]. If a function scales as $G\left(\lambda^{\alpha_{1}} x_{1}, \lambda^{\alpha_{2}} x_{2}, \lambda^{\alpha_{3}} x_{3}\right)=\lambda^{\eta} G$, then

$$
\eta G=\alpha_{1} \frac{\partial G}{\partial x_{1}} x_{1}+\alpha_{2} \frac{\partial G}{\partial x_{2}} x_{2}+\alpha_{3} \frac{\partial G}{\partial x_{3}} x_{3} .
$$

Renaming $G=\tilde{\Lambda}, x_{1}=\tau, x_{2}=S, x_{3}=R^{D-2}$, one has $\eta=2 /(2-D), \alpha_{1}=c$ and $\alpha_{2}=\alpha_{3}=1$. With this we obtain

$$
\tilde{\Lambda}=\frac{2-D}{2}\left(c \frac{\partial \tilde{\Lambda}}{\partial \tau} \tau+\frac{\partial \tilde{\Lambda}}{\partial S} S+\frac{\partial \tilde{\Lambda}}{\partial R^{D-2}} R^{D-2}\right) .
$$

If $c=0$, there is no phase transition [17. This is unexpected, considering Hawking-Page theory, and so we discard this case.

The general solution for $c \neq 0$ of the differential equation (38) is

$$
\tilde{\Lambda}=\tau^{-\frac{2}{D-2} \frac{1}{c}} \mathcal{F}(z, y), \quad z=S \tau^{-\frac{1}{c}}, \quad y=R^{D-2} \tau^{-\frac{1}{c}},
$$


where $\mathcal{F}$ is any homogeneous function of order zero, as is clear from the definition of its arguments above. Equation (39) fixes the functional form of the cosmological constant in the thermodynamic description, solely on the grounds of the homogeneity condition.

We also observe that homogeneity relations (36) impose limitations on the possible choices of $\varepsilon_{0}$, restricting it to specific combinations of the thermodynamic quantities. These combinations need to be homogeneous of order $1 /(2-D)$ and, by the Euler theorem, $\varepsilon_{0}$ must has the form

$$
\varepsilon_{0}=\frac{1}{R} \mathcal{G}(z, t), \quad z=R \tau^{\frac{1}{c} \frac{1}{(2-D)}}, \quad t=R S^{\frac{1}{2-D}},
$$

where $\mathcal{G}$ is some arbitrary homogeneous function of order 0 . Concerning the dependence of $\varepsilon_{0}$ on $z$ and $t$, it should be taken into account that the cosmological constant is a function on phase space, and so $\varepsilon_{0}$ depends at this point on $\tau$ and $S$ implicitly through $\Lambda$ (besides the explicit dependence on $R$ ). Another result is that one can obtain the Smarr formula [13, 33, 29] by using Euler's formula (37). Setting $G=E, x_{1}=S, x_{2}=R^{D-2}, x_{3}=\tau$, we have

$$
E=\frac{D-2}{D-3}\left(T_{L} S-B_{D} P R^{D-2}+c \tau \xi\right), \quad \xi=\frac{\partial E}{\partial \tau} .
$$

Thus, the constant $c$ can be understood as a measure of the contribution to the energy that arises due to the extension of phase space by the canonical pair $(\tau, \xi)$. The $R$-dependence in (41) implies that the energy is dependent on the position of the observer at the boundary. In the same way, if the AdS "box" increases or decreases in size (i.e., a change in the AdS radius $l=1 / \sqrt{-\tilde{\Lambda}}$ ), it will change the spacetime energy by an amount weighted by the constant $c$.

\subsection{Fixing the Thermodynamics}

The function $\mathcal{F}$ in (39) can be determined by demanding that the cosmological constant is fixed by geometric arguments. Let us consider the relation that determines the horizon radius, $N\left(r_{+}\right)=0$, or more explicitly,

$$
1-\frac{16 \pi M}{(D-2) B_{D}} \frac{1}{r_{+}^{D-3}}-\frac{2 \Lambda}{(D-1)(D-2)} r_{+}^{2}=0 .
$$

There are three parameters in this equation, and hence $r_{+}$and $\Lambda$ can be chosen arbitrarily, as long as $r_{+}$is positive and $\Lambda$ is negative. Any choice of $r_{+}>0$ and $\Lambda<0$ generates a Schwarzschild-AdS geometry. Since the entropy $S=$ $B_{D} r_{+}^{D-2} / 4$ is a function of $r_{+}$alone, any pair $(S, \Lambda)$ with $S>0$ and $\Lambda<0$ is associated with a SAdS spacetime, with $S$ and $\Lambda$ independent. Also, a particular choice of a surface $r=R$ for the definition of the quasilocal quantities should not depend on the background geometry (characterized by $r_{+}$and $\Lambda$, or by $S$ and $\Lambda$ ). Therefore, geometric arguments suggest that thermodynamic variable $\Lambda$ should be independent of $S$ and $R$. From (39) we see that the only way to satisfy these requirements is if $\mathcal{F}=-K$ is constant, so

$$
\tilde{\Lambda}=-K \tau^{-\frac{2}{D-2} \frac{1}{c}} \text {. }
$$


Taking (43) into account, $d E=\left.\tilde{\theta}\right|_{\{\phi\}=0}$, where $\{\phi\}$ is the total set of constraints, is expressed as

$$
\begin{aligned}
d E & =\frac{B_{D}}{4 \pi}(p d q+\varpi d x) \\
& -\left[\frac{B_{D}(D-2)}{16 N \pi}\left(q^{\frac{D-1}{D-2}}-x^{\frac{D-1}{D-2}}\right) \frac{\partial \tilde{\Lambda}}{\partial \tau}+x B_{D} \frac{\partial \varepsilon_{0}}{\partial \tau}\right] d \tau,
\end{aligned}
$$

and the additional constraint (32) becomes

$$
\phi_{3}=\xi+\frac{B_{D} K \tau^{-\frac{2}{D-2} \frac{1}{c}-1}}{8 N c \pi}\left(q^{\frac{D-1}{D-2}}-x^{\frac{D-1}{D-2}}\right)+x B_{D} \frac{\partial \varepsilon_{0}}{\partial \tau} .
$$

One observes that the first law of Thermodynamics is given by eq. (44) evaluated at the constraint surface $\phi_{1}=\phi_{2}=\phi_{3}=0$. The equation $\phi_{3}=0$ becomes, in thermodynamic variables,

$$
\xi=-\frac{2}{D-2} \frac{1}{N c \tau} \frac{\Lambda}{8 \pi}\left[\theta(R)-\theta\left(r_{+}\right)\right]-B_{D} R^{D-2} \frac{\partial \varepsilon_{0}}{\partial \tau},
$$

where

$$
\theta(r)=\frac{B_{D}}{D-1} r^{D-1}
$$

is the Euclidian volume of a sphere of radius $r$ in $D$ dimensions. Since $\Lambda / 8 \pi$ can be interpreted as the (volumetric) energy density of the AdS space, it follows that, for $\varepsilon_{0}=0, c N \xi \tau$ is proportional to the energy of the spacetime inside the radius $R$ sphere discounted the volume $\theta\left(r_{+}\right)$that, as discussed in section 3. can be identified as the volume extracted from spacetime due to the presence of the black hole. That is the only quantity with physical meaning. By substituting (46) in (41), we obtain a Smarr formula in terms of $R, S$ and $\Lambda$,

$$
\begin{aligned}
\frac{D-3}{D-2} E & =T_{L} S-\frac{B_{D} \tilde{\Lambda}}{8 \pi N}\left[R^{D-1}-\left(\frac{4 S}{B_{D}}\right)^{\frac{D-1}{D-2}}\right]-B_{D} R^{D-2}\left(P+c \tau \frac{\partial \varepsilon_{0}}{\partial \tau}\right) \\
& =T_{L} S-\frac{2 \Lambda}{8 \pi} \frac{\theta(R)-\theta\left(r_{+}\right)}{(D-2) N}-B_{D} R^{D-2}\left(P+c \tau \frac{\partial \varepsilon_{0}}{\partial \tau}\right) .
\end{aligned}
$$

The second expression in eq. (48) can be compared with the analogous result in [13]. The choice of a specific value of $c$ is a matter of convenience. As shown in [17, it is not possible to use $\Lambda$ as a thermodynamic variable in the simple extension of the minimal SAdS Thermodynamics. This is because the conjugate variable $\xi$ will not depend on $\tau$ and the Legendre transformation between the pair (necessary to construct the internal energy from the enthalpy) is singular [18, 51]. However, in the quasilocal approach discussed here, it is clear from (32) that, even if $\tau=\Lambda, \xi$ still depends on $\tau$ (or $\Lambda$ ) through the lapse function $N(S, R, \tau)$ in the denominator of the first term in the right hand side of (32), even though the derivative $\partial \Lambda / \partial \tau$ is constant. So, in the present setup, 
it is possible to promote $\Lambda$ to a thermodynamic variable without introducing singularities. This choice corresponds to

$$
c=-\frac{2}{D-2} .
$$

By setting $K=16 \pi /(D-1)(D-2)$ in (43), we have that $\tau=|\Lambda| / 8 \pi$ is the energy density of spacetime, as in Dolan's original proposal [15, 18]. Besides, for $\varepsilon_{0}=0$, the conjugate variable $\xi=\theta(R)-\theta\left(r_{+}\right)$is the total geometric volume of the local description, discounted the black hole volume (taking into account the correction by the lapse function). Or, in other words, $\xi$ is the total geometric volume measured by an observer characterized by $N=1$. We stress that, with the treatment presented here, it is possible to promote $\Lambda$ to a thermodynamic variable, as was originally proposed in [33, 34, 26, 18.

Although interesting, there is nothing fundamental in the choice of $c$ in (49) from the thermodynamic point of view. A better strategy might be to use the arbitrariness in $c$ in order to simplify a specific problem. For example, one can choose to work with the fundamental equation $U(S, R, \xi)=E-\xi \tau$,

$$
U=(D-2)\left\{\frac{B_{D}}{8 \pi N}\left[\left(\frac{4 S}{B_{D}}\right)^{\frac{D-3}{D-2}}-R^{D-3}\right]-\left[c+\frac{1}{D-2}\right] \tau \xi\right\} .
$$

In this case, the expression can be simplified by setting

$$
c=-\frac{1}{D-2} \text {. }
$$

That is, using $\sqrt{-\Lambda}$ as a thermodynamic variable instead of $\Lambda$. With this choice

$$
U=(D-2) \frac{B_{D}}{8 \pi N}\left[\left(\frac{4 S}{B_{D}}\right)^{\frac{D-3}{D-2}}-R^{D-3}\right] .
$$

The choice (51) has the additional advantage that, as in the usual thermodynamics, all the variables can be classified in two types: the extensive variables $\left(S, B_{D} R^{D-2}, \xi\right)$, of degree one, and the intensive variables $(T, P, \tau)$, of degree $1 /(2-D)$. We note that, in this case, $\xi$ is an extensive variable, so $E$ is a function of two extensive and one intensive variable, and could be interpreted as the enthalpy (as proposed in [15, 18, for the extension of the minimal setup). In addition, $U$ depends only on the extensive variables and can be identified with the internal energy (a quantity ill-defined in [15, 18]). Other choices of $c$ could prove to be more convenient for different thermodynamic potentials.

Some more comments about the choice of $c$ can be made. The first law of Thermodynamics (44) alone cannot fix the parameter $c$, since it only fixes the homogeneity of the product $\xi d \tau$. And according to the homogeneity relations eq. (36), this product will be independent of $c$. In fact, the only physical quantity independent of $c$ is the product $\tau \xi$. The physical meaning of variables $(\tau, \xi)$ can only be seen after the fixation of $c$. One example was discussed in this section, 
eq. (51), with $\tau=1 / l$. Another example is the case $c=(D-1) /(D-2)$, where $\tau$ can be seen as the effective volume in the anti de Sitter space [52].

It should be noted that the asymptotic behavior of the theory depends heavily on the choice of the arbitrary function $\varepsilon_{0}$, as it was pointed out in [19]. In our treatment, the only restriction upon the $\varepsilon_{0}$ function is that it must obey eq. (40). The asymptotic behavior of $P$ in eq. (26) and $\xi$ in eq. (46) changes with different choices of $\varepsilon_{0}$, but once we fix it there is no ambiguity.

Besides completely defining the Thermodynamics in terms of the parameters $\Lambda, R$ and $r_{+}$, the above results guarantee the homogeneity condition and a singularity-free Thermodynamics. As a consequence, any thermodynamic potential can be used in this description (e.g., the enthalpy, the Helmholtz free energy and so on), since generic Legendre transformations linking these potentials are possible, as in Thermodynamics of non-gravitational systems.

\section{Stability conditions}

In this section we analyze the stability conditions for the system. We have a consistent thermodynamic description for the SAdS black hole, characterized by the equations of state for $P$ and $T_{L}$ in (26) and (27) respectively. In addition, there is the constraint (45):

$$
\xi=\frac{B_{D} K(D-2)}{16 \pi N}\left[\left(\frac{4 S}{B_{D}}\right)^{\frac{D-1}{D-2}}-R^{D-1}\right]-R^{D-2} B_{D} \frac{\partial \varepsilon_{0}}{\partial \tau} .
$$

In (53) we considered the fixed value (49) for the constant $c$. We stress that there is nothing special with this choice and one could work with the general expression (46).

Let us consider the heat capacity

$$
C_{R, \Lambda}=\left(\frac{\partial E}{\partial T_{L}}\right)_{R, \Lambda}=T_{L}\left(\frac{\partial S}{\partial T_{L}}\right)_{R, \Lambda},
$$

since our system has three degrees of freedom, and we consider processes with two variables fixed. From (27), it follows that

$$
C_{R, \Lambda}=-(D-2) \frac{S}{v}\left[D-3-\tilde{\Lambda}(D-1)\left(\frac{4 S}{B_{D}}\right)^{\frac{2}{D-2}}\right],
$$

where

$$
v(S, R, \Lambda)=D-3+\tilde{\Lambda}(D-1)\left(\frac{4 S}{B_{D}}\right)^{\frac{2}{D-2}}-\frac{8 \pi^{2} T_{L}^{2}}{R^{D-3}}\left(\frac{4 S}{B_{D}}\right)^{\frac{D-1}{D-2}} .
$$

The limit $R \rightarrow \infty$ of the function $v(S, R, \Lambda)$ is

$$
\lim _{R \rightarrow \infty} v=D-3+\tilde{\Lambda}(D-1)\left(\frac{4 S}{B_{D}}\right)^{\frac{2}{D-2}} .
$$


Therefore, as $R$ grows, the heat capacity diverges as $v \rightarrow 0$, when

$$
\tilde{\Lambda} \frac{D-3}{D-1}=-\left(\frac{4 S}{B_{D}}\right)^{\frac{2}{D-2}} .
$$

In terms of the horizon radius $r_{+}$and the the cosmological constant $\Lambda$, the previous equation assumes the form

$$
r_{+}=r_{0}, \quad r_{0} \equiv \sqrt{\frac{(D-3)(D-2)}{2|\Lambda|}} .
$$

We define a temperature $T_{S L}$ which is the quasilocal temperature (27) taken at the critical radius (59), which coincides with the minimum of the temperature $T_{L}$ in the large $R$ limit. Thus, if the temperature at the boundary is set to a value smaller than $T_{S L}$, no black hole can exist, and the system will be dominated by thermal radiation in an anti de Sitter background. On the other hand, if the temperature is set to a value greater than $T_{S L}$, there will be two solutions. Namely, a larger and stable black hole, with $r_{+}>r_{0}$ and positive heat capacity; and a smaller and unstable black hole, with $r_{+}<r_{0}$ and negative heat capacity. The temperature $T_{S L}$ is the transition temperature for the Small-Large black hole transition [53.

Therefore, SAdS black holes can be in thermal equilibrium within an environment with a negative cosmological constant when $R \rightarrow \infty$. This was originally shown by Hawking and Page [53] using a Euclidean path integral approach. It is important to note that the Small-Large (SL) black hole phase transition is obtained from the minimum of the Hawking temperature (8) which, in general, is different from the minimum of the local temperature $T_{L}$. The analysis we have made here is possible only in the large $R$ limit, when the two minima asymptotically coincide and, thus, one can read the SL phase transition from the temperature $T_{L}$.

We also note that, for the $D=3 \mathrm{BTZ}$ black hole, $v$ is independent of $R$ and a straightforward calculation shows that $C_{R, \Lambda}=S$, which is always positive. Thus, our description for the BTZ black hole has no local instability, in agreement with [51].

\section{Conclusions and perspectives}

The first attempts at describing the thermodynamic behavior of SAdS black holes were not entirely consistent [17. The minimal description lacks homogeneity and the temperature is not well-defined, since the surface gravity is not uniquely defined. The latter issue can be treated using quasilocal quantities defined for $(D-2)$-spheres [19, 20, 49. With this, one obtains a theory in which the temperature of the black hole depends explicitly on the position of the observer.

However, the quasilocal formalism does not fix the homogeneity issue. We solve this problem by using a Hamiltonian approach to Thermodynamics presented in [22]. We are able to find a new thermodynamic theory specifying the 
entropy $S$, the observer position $R$ and the cosmological constant $\Lambda$. We are also able to give an explicit form for the Smarr formula in terms of these quantities. This result shows how the geometry contributes to the internal energy of the thermodynamic description. It has all the properties of the previously descriptions (i.e., has a well-defined temperature) and furthermore is homogeneous. As a result, unlike all previous descriptions, the presented development allows the use of any thermodynamic potential, which can be obtained via Legendre transformations. Our approach brings the Thermodynamics of Schwarzchild-anti de Sitter black holes closer to the description of standard thermodynamic systems.

Besides predicting Small-Large black hole phase transitions, new effects are expected, due to the existence of heat capacities associated to different kinds of processes, as well as other equations of state which give analogs of the compressibility coefficients of the standard theory. A natural development of the present work would be the characterization of phase transitions and critical exponents. Investigation along those lines is currently under way.

\section{Acknowledgements}

W. B. F. acknowledges the support of the Coordination for the Improvement of Higher Education Personnel (CAPES), Brazil with grants \#1591255 and \#1775078. C. M. is supported by grant \#2015/24380-2, São Paulo Research Foundation (FAPESP), Brazil; and grants \#307709/2015-9 and \#420878/20165, National Council for Scientific and Technological Development (CNPq), Brazil. R.F. acknowledges the support of São Paulo Research Foundation (FAPESP), Brazil, with grant \#2016/03319-6.

\section{References}

[1] T. Padmanabhan Rep. Prog. Phys. (2010) 73046901 (arXiv:0911.5004)

[2] T. Jacobson Phys. Rev. Lett. (1995) 751260 (arXiv:9504004)

[3] T. Padmanabhan Current Science (2015) 1092236 (arXiv:1512.06546)

[4] J.D. Bekenstein Phys. Rev. D (1973) 72333

[5] J.M. Bardeen, B. Carter, S.W. Hawking Commun. Math. Phys. (1973) 31 161

[6] S.W. Hawking Commun. Math. Phys. (1975) 43199

[7] R.M. Wald Living Rev. Rel. (2001) 46 (arXiv:9912119v2)

[8] D. Kubiznak, R.B. Mann, M. Teo Class. Quant. Grav. (2017) 34063001 (arXiv:1608.06147)

[9] J. Maldacena Adv. Theor. Math. Phys. (1998) 2231 (arXiv:9711200) 
[10] E. Witten Adv. Theor. Math. Phys. (1998) 2253 (arXiv:9802150)

[11] S.S. Gubser, I.R. Klebanov, A.M. Polyakov Phys. Lett. B (1998) 428105 (arXiv:9802109)

[12] H.A. Buchdal Concepts on classical thermodynamics (2009) (Cambridge: Cambridge University Press)

[13] D. Kastor, S. Ray, J. Traschen Class. Quant. Grav. (2009) 26195011 (arXiv:0904.2765)

[14] C.V. Johnson Class. Quant. Grav. (2014) 31205002 (arXiv:1404.5982)

[15] B.P. Dolan Where Is the PdV in the First Law of Black Hole Thermodynamics?, Open Questions in Cosmology (2012) (G.J. Olmo ed., InTech) (arXiv:1209.1272)

[16] F. Belgiorno J. Math. Phys. (2003) 441089 (arXiv:0210021)

[17] M.C. Baldiotti, R. Fresneda, C. Molina Ann. Phys. (2017) 38222 (arXiv:1701.01119)

[18] B.P. Dolan Class. Quant. Grav. (2011) 28235017 (arXiv:1106.6260)

[19] J.D. Brown, J. Creighton, R.B. Mann Phys. Rev. D (1994) 506394 (arXiv:9405007)

[20] J.D. Brown, J.W. York Jr Phys. Rev. D (1993) 471407 (arXiv:9209012)

[21] R.C. Tolman Phys. Rev. (1930) 35904

[22] M.C. Baldiotti, R. Fresneda, C. Molina Ann. Phys. (2016) 373245 (arXiv:1604.03117)

[23] S.G. Rajeev Ann. Phys. (2008) 32322652285 (arXiv:0711.4319v2)

[24] T. Padmanabhan Phys.Rept. (2005) 40649 (arXiv:0311036)

[25] M. Natsuume AdS/CFT User Guide (2016) (Springer) (arXiv:1409.3575)

[26] C. Teitelboim Phys. Lett. B (1985) 158293

[27] J.D. Brow, C. Teitelboim Nucl. Phys. B (1988) 297787

[28] J.D.E. Creighton, R.B. Mann Phys. Rev. D (1995) 524569 (arXiv:9505007)

[29] L. Smarr Phys. Rev. Lett. (1973) 30521

[30] J.P. Gauntlett, R.C. Myers, P.K. Townsend Class. Quant. Grav. (1999) 16 1 (arXiv:9810204)

[31] P.K. Townsend, M. Zamaklar Class. Quant . Grav. (2001) 185269 (arXiv:0107228) 
[32] M.M. Caldarelli, G. Cognola, D. Klemm Class. Quant. Grav. (2000) 17399 (arXiv:9908022)

[33] Y. Sekiwa Phys. Rev. D (2006) 73084009 (arXiv:0602269)

[34] M. Urano, A. Tomimatsu, H. Saida Class. Quant. Grav. (2009) 26105010 (arXiv:0903.4230)

[35] S. Wang, S.Q. Wu, F. Xie, L. Dan Chin. Phys. Lett. (2006) 231096 (arXiv:0601147)

[36] G.L. Cardoso, V. Grass Nucl. Phys. B (2008) 803209 (arXiv:0803.2819)

[37] J.H. Traschen Phys. Rev. D (1985) 31283

[38] J.H. Traschen, D. Fox Class. Quant. Grav. (2004) 21289 (arXiv:0103106)

[39] D. Sudarsky, R.M. Wald Phys. Rev. D (1992) 461453

[40] F. Belgiorno Phys. Lett. A (2003) 312324 (arXiv:0210020)

[41] S.L. Bazanski, P. Zyla Gen. Rel. Grav. (1990) 22379

[42] D. Kastor Class. Quant. Grav. (2008) 25175007 (arXiv:0804.1832)

[43] D. Kastor, S. Ray, J. Traschen Class. Quant. Grav. (2010) 27235014 (arXiv:1005.5053)

[44] L.F. Abbott, S. Deser Nucl. Phys. B (1982) 19576

[45] D. Kubiznak, R.B. Mann J. High Energ. Phys. (2012) 1207033 (arXiv:1205.0559)

[46] D. Kubiznak, R.B. Mann Can. J. Phys. (2015) 93999 (arXiv:1404.2126)

[47] R.B. Mann Springer Proc. Phys. (2016) 170197

[48] G. Barnich, G. Compre J. Math. Phys. (2008) 49042901 (arXiv:0708.2378)

[49] J.W. York Found. Phys. (1986) 16249

[50] R. Arnowitt, S. Deser, C.W. Misner Gen. Rel. Grav. (2008) 401997 (arXiv:0405109)

[51] B.P. Dolan Class. Quant. Grav. (2011) 28125020 (arXiv:1008.5023)

[52] W.S. Elias, C. Molina, M.C. Baldiotti Phys. Rev. D (2019) 99084028 (arXiv:1803.05921)

[53] S.W. Hawking, D.N. Page Commun. Math. Phys. (1983) 87577 